Check for updates

Cite this: J. Mater. Chem. A, 2019, 7, 24771

Received 28th August 2019 Accepted 7th October 2019

DOI: $10.1039 /$ c9ta09500d

rsc.li/materials-a

\section{Green radicals of potassium poly(heptazine imide) using light and benzylamine $\uparrow$}

\author{
Yevheniia Markushyna, $\ddagger^{a}$ Paolo Lamagni,,$^{\text {abc }}$ Christian Teutloff, ${ }^{d}$ \\ Jacopo Catalano, (D) c Nina Lock, ${ }^{\text {bc }}$ Guigang Zhang, (D) a Markus Antonietti (D) a \\ and Aleksandr Savateev (iD) *a
}

Tinted long-lived ionic carbon nitride radicals were recently introduced and applied in photocatalysis and energy storage. However, the reason for their higher activity in the photocatalytic reaction and optimal conditions for generating such radicals remain vague. Herein, we study the conditions for carbon nitride photocharging to achieve a higher charge density and validate a convenient method to quantify the number of electrons accumulated in carbon nitride semiconductors by quenching its radicals with methylviologen in the dark. In the presence of $\mathrm{CO}_{2}$, potassium poly(heptazine imide) (K-PHI) can be charged by up to $1000 \mu \mathrm{mol}$ of electrons per gram of the material using benzylamine as an electron donor. Under the same conditions, mesoporous graphitic carbon nitride can accumulate only $50 \mu \mathrm{mol}$ of electrons per gram. The products of the benzylamine oxidative coupling are imine and ammonia.

\section{Introduction}

Photocatalysis has become a topical field of research in organic chemistry starting from the beginning of $21^{\text {st }}$ century due to its simple reaction conditions and omnitude. ${ }^{1}$ Indeed, the same photocatalyst can promote different chemical reactions. $^{2,3}$ Due to their chemical stability, ease of preparation, structural tunability and availability, carbon nitrides became convenient catalysts in organic synthesis. ${ }^{4-7}$ Moreover,

${ }^{a}$ Max-Planck Institute of Colloids and Interfaces, Department of Colloid Chemistry, Research Campus Golm, 14476 Potsdam, Germany. E-mail: oleksandr.savatieiev@ mpikg.mpg.de

${ }^{b}$ Carbon Dioxide Activation Center (CADIAC), Aarhus University, Interdisciplinary Nanoscience Center (iNANO), Department of Chemistry, DK-8000 Aarhus C, Denmark.E-mail: nlock@eng.au.dk

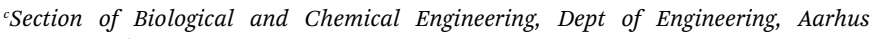
University, Åbogade 40, DK-8200 Aarhus N, Denmark

${ }^{d}$ Freie Universität Berlin, Department of Physics, 14195 Berlin, Germany

$\dagger$ Electronic supplementary information (ESI) available: Catalyst preparation procedure and characterization data; ${ }^{1} \mathrm{H},{ }^{13} \mathrm{C}$ and ${ }^{15} \mathrm{~N}$ NMR spectra; supplementary figures. See DOI: 10.1039/c9ta09500d

$\$$ These authors contributed equally to this work. poly(heptazine imides) are capable of photocharging. ${ }^{8}$ Fig. 1 sketches the mechanism of IDEAS (Illumination-Driven Electron Accumulation in Semiconductors) - a capacious acronym that we propose for this process in the present communication.

On the energy scale, it can be schematically explained as follows. By absorbing a photon, potassium poly(heptazine imide) (K-PHI) is converted to the excited state (K-PHI*). Electron transfer from the HOMO of the electron donor (ED) lying above the valence band (VB) of K-PHI reduces $\mathrm{K}-\mathrm{PHI}$ * and gives a negatively charged radical anion (K-PHI ${ }^{-}$). IDEAS is accompanied by a distinct color change of K-PHI and related materials from yellow to green or blue. ${ }^{8-11}$ The energy stored in K-PHI is then released by exposing $\mathrm{K}_{-} \mathrm{PHI}^{-}{ }^{-}$to the electron acceptors (EAs). This step leads to the recovery of K-PHI and formation of



Fig. 1 Schematic representation of IDEAS as exemplified for K-PHI. Image of $\mathrm{K}-\mathrm{PHI}$ suspension in acetonitrile in the presence of benzylamine under a protective $\mathrm{Ar}$ atmosphere before light irradiation (yellow) and after light irradiation (green). Abbreviations: VB - valence band; $C B$ - conduction band; ED - electron donor; EA - electron acceptor; and $\mathrm{EA}_{\text {red }}$ - reduced electron acceptor. 
a reduced electron acceptor $\left(\mathrm{EA}_{\text {red }}\right)$. K-PHI can accumulate up to several hundred $\mu \mathrm{mol}$ of electrons per gram of the material., ${ }^{\mathbf{9 1 2}}$

Due to high reactivity of $\mathrm{K}-\mathrm{PHI}^{-}$, characterization of this material is a challenging task. Nevertheless, transient absorption spectroscopy has been used to characterize the green radicals of cyanamide-functionalized poly(heptazine imide). ${ }^{\mathbf{1 3 , 1 4}}$

Earlier, we have shown superior activity of K-PHI in comparison with covalent carbon nitrides in reductive cyclocondensation of chalcones to cyclopentanoles, ${ }^{\mathbf{1 0}}$ synthesis of $\mathrm{N}$ fused pyrroles ${ }^{\mathbf{1 5}}$ and oxidative thiolation of toluene. ${ }^{9}$ On the other hand, tinted long-lived carbon nitride radicals have been applied in $\mathrm{H}_{2}$ production ${ }^{8,16}$ and $\mathrm{O}_{2}$ reduction to $\mathrm{H}_{2} \mathrm{O}_{2} \cdot{ }^{11,17}$ Based on cyanamide-functionalized poly(heptazine imide), Lotsch et al. designed a "solar battery" that combines in one device a light harvester and a charge storage reservoir. ${ }^{18}$

While already plentiful chemistry and unusual properties make K-PHI a promising material to address other synthetic challenges, a deeper understanding of the IDEAS mechanism is required to use carbon nitrides more efficiently in lightconversion applications.

It should be mentioned that the electron capacity of a relevant carbon nitride material, cyanamide-functionalized poly(heptazine imide), has been investigated electrochemically. ${ }^{18}$ However, related to the application in organic photocatalysis by suspending a semiconductor particulate in an organic solvent, a more convenient method for measuring the electron capacity of carbon nitride would comprise a redox indicator. Such a method would not require an electrolyte and a specialized electrochemical setup, while quenching of the reaction mixture with a suitable redox indicator would allow for quasi in situ quantification of IDEAS.

Herein, we develop and validate a convenient method to quantify IDEAS using benzylamine as a model electron donor and methylviologen as a redox indicator. We compare the capacity of K-PHI and covalent mesoporous graphitic carbon nitride (mpg-CN) under $\mathrm{Ar}$ and $\mathrm{CO}_{2}$.

\section{Results and discussion}

K-PHI was characterized using different techniques and the summary is given in Fig. S2 and S3. $\dagger^{+19}$ The number and position of diffraction peaks in the PXRD pattern are identical to the ones reported earlier (Fig. S2a $\dagger$ ). ${ }^{\mathbf{2 0 , 2 1}}$ The valence band maximum (VBM) in K-PHI determined by ultraviolet photoelectron spectroscopy (UPS) is located at $\sim 2.5 \mathrm{~V}$ (Fig. S2e $\dagger$ ). K$\mathrm{PHI}$ has the main onset of absorption at $460 \mathrm{~nm}$ related to $\pi-\pi^{*}$ transitions and a band of lower intensity with the edge at 650 $\mathrm{nm}$ (n- $\pi^{*}$ transitions) (Fig. S $\left.2 \mathrm{f}^{\dagger}\right)$. By using the $\pi-\pi^{*}$ band, an optical band gap of $2.72 \mathrm{eV}$ was determined. K-PHI shows maximum fluorescence at $\sim 500 \mathrm{~nm}$ upon sample excitation with $350 \mathrm{~nm}$ (Fig. S2g porosity as evidenced by the $\mathrm{N}_{2}$ sorption isotherm (Fig. $\mathrm{S} 2 \mathrm{~h} \dagger$ ). The specific surface area is $89 \mathrm{~m}^{2} \mathrm{~g}^{-1}$. FT-IR shows peaks typical for K-PHI and carbon nitrides in general: $800 \mathrm{~cm}^{-1}$ (heptazine out-of-plane bending), $916 \mathrm{~cm}^{-1}$ and $985 \mathrm{~cm}^{-1}$ (symmetric vibrations of the metal- $\mathrm{NC}_{2}$ moiety), $1116-1390 \mathrm{~cm}^{-1}$ (C-O stretch), $1574 \mathrm{~cm}^{-1}$ (secondary amine $\mathrm{N}-\mathrm{H}$ bend), $1653 \mathrm{~cm}^{-1}$ (primary amine $\mathrm{N}-\mathrm{H}$ bend), $2184 \mathrm{~cm}^{-1}$ (nitrile stretch), and 2500-3600 $\mathrm{cm}^{-1}$ (N-H stretch) (Fig. S2i ( $^{22}$ In water, K-PHI breaks into particles of $c a .50$ to $200 \mathrm{~nm}$ in diameter with an average size of $94 \mathrm{~nm}$ (Fig. $\mathrm{S} 2 \mathrm{j} \dagger$ ). The ordered structure of the catalyst is readily observed in the high resolution transmission electron microscopy (HR-TEM) image of K-PHI (Fig. S2l $\dagger$ ). Relaxation of the excited state via a radiative path occurs within 0.66 ns (Fig. S3†).

In order to quantify the number of electrons accumulated in $\mathrm{K}-\mathrm{PHI}$, we performed a series of experiments. $\mathrm{K}-\mathrm{PHI}^{-}{ }^{-}$was generated in situ using benzylamine as an electron donor followed by the addition of methylviologen dichloride $\left(\mathrm{MV}^{2+}\right)$. The latter was reduced via a one-electron process to a radical cation $\left(\mathrm{MV}^{\cdot+}\right)$ that has a pronounced blue colour. The amount of $\mathrm{MV}^{\cdot+}$ produced upon $\mathrm{K}^{-} \mathrm{PHI}^{\cdot-}$ quenching with $\mathrm{MV}^{2+}$ was determined measuring the solution absorbance at $600 \mathrm{~nm}$ (Fig. S4 $\dagger$ ). The amount of $\mathrm{MV}^{\cdot+}$ is therefore equal to the amount of electrons accumulated in the catalyst. Fig. 2a summarizes this procedure.

Using the abovementioned methodology, the electron capacity of mpg-CN was determined to be $43 \pm 5 \mu \mathrm{mol} \mathrm{g}{ }^{-1}$, however no apparent colour change was observed (Fig. 2b). The higher surface area of mpg-CN compared to K-PHI, i.e. $180 \mathrm{~m}^{2}$ $\mathrm{g}^{-1}$ versus $89 \mathrm{~m}^{2} \mathrm{~g}^{-1}$, apparently is not a decisive factor for efficient IDEAS. Instead, the crystalline structure and the ability of the material to stabilize negative charges observed for K-PHI are important. It has also been reported that cyanamide groups in poly(heptazine imide) are responsible for electron accumulation. ${ }^{\mathbf{8} 21}$ In the case of K-PHI, a deeper reaction mixture colour was observed in the presence of $\mathrm{CO}_{2}$ (the interaction between $\mathrm{CO}_{2}$ and benzylamine is investigated below), which correlates with a higher electron capacity of $957 \pm 82 \mu \mathrm{mol} \mathrm{g}{ }^{-1}$ compared to $701 \pm 46 \mu \mathrm{mol} \mathrm{g}^{-1}$ under Ar. Nevertheless, the electron capacity of K-PHI under Ar using benzylamine as an electron donor is still 5.8 times higher compared to that using benzyl alcohol as an electron donor. ${ }^{9}$ This illustrates the much better ability of amines to quench reductively the excited state of the carbon nitride photocatalyst. In the control experiment without the carbon nitride photocatalyst, $\mathrm{MV}^{2+}$ is not reduced by benzylamine alone.

Fig. 2c illustrates the effect of the benzylamine concentration, i.e. the electron donor concentration, on the number of electrons accumulated in K-PHI. K-PHI reaches saturation at a benzylamine concentration of $60 \mu \mathrm{mol} \mathrm{mL}{ }^{-1}$, at which the electron capacity approaches $957 \pm 82 \mu \mathrm{mol} \mathrm{g}{ }^{-1}$. This number corresponds to $92 \pm 8 \mathrm{C} \mathrm{g}^{-1}$ and is higher than that reported for cyanamide-functionalized poly(heptazine imide) determined from electrochemical discharge profiles $\left(45 \mathrm{C} \mathrm{g}^{-1}\right){ }^{18}$ Given that the molecular weight of a K-PHI monomer unit is $223 \mathrm{~g} \mathrm{~mol}^{-1}$ (Fig. S5 $\dagger$ ), ${ }^{23}$ it can be concluded that every $4^{\text {th }}$ heptazine unit in K-PHI bears an uncoupled electron, while for cyanamide-functionalized poly(heptazine imide) one electron is being stored on every $9^{\text {th }}$ and $10^{\text {th }}$ cyanamide-heptazine motif. ${ }^{8,18}$ Taking into account that K-PHI is represented by particles with an average diameter of $100 \mathrm{~nm}$, the volume-tosurface ratio of heptazine units is 26 . In other words, in order to achieve such a high density of trapped electrons, they must be stored not only on the surface of the material, but to be 
a)

d) b)
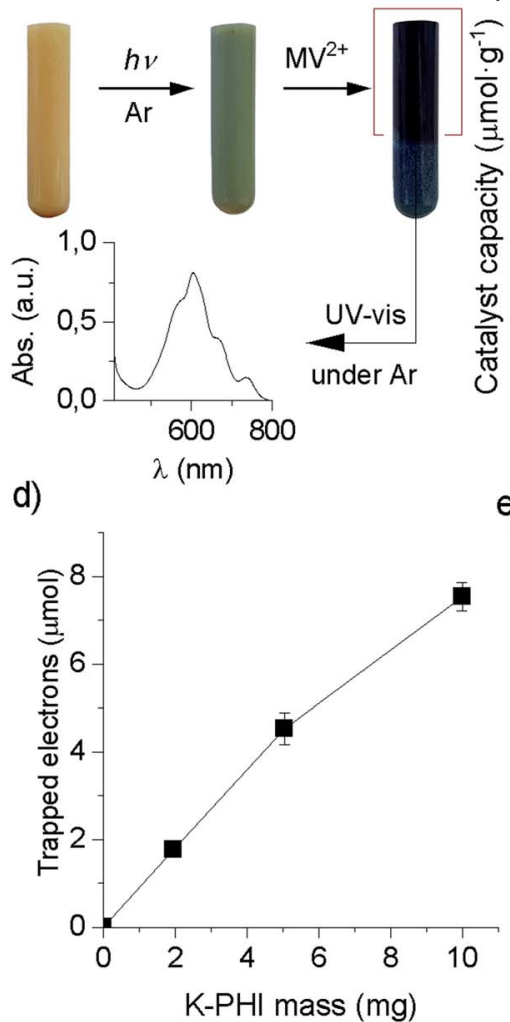

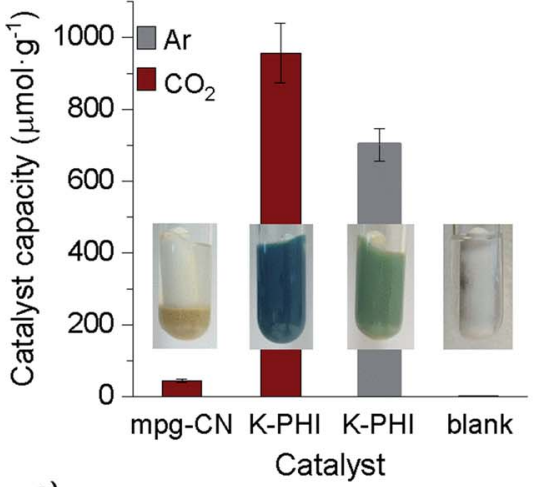

e)



c)

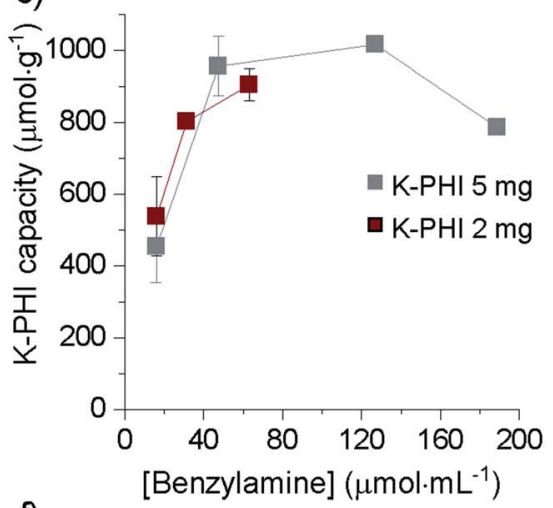

f)

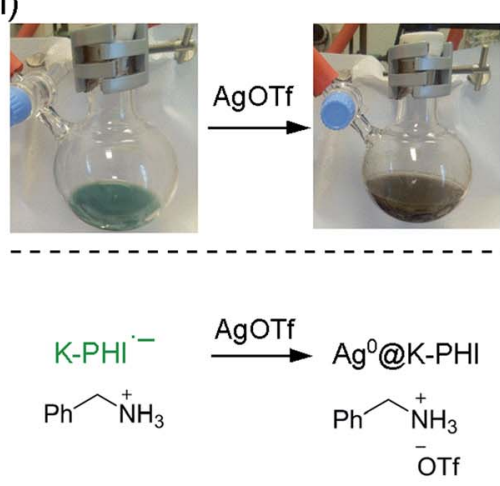

Fig. 2 Insights into carbon nitride charge storage capacity. (a) Visual appearance of the reaction mixture - K-PHI, benzylamine, acetonitrile, under $\operatorname{Ar}(1$ bar). From left to right: reaction mixture (under Ar) before light irradiation (yellow), after light irradiation (green), and methyl viologen $\left(\mathrm{MV}^{2+}\right)$ addition (blue). Schematic representation of the procedure for measuring the absorption of a $\mathrm{MV}^{\cdot+}$ solution; (b) electron capacity of carbon nitrides and appearance of the reaction tubes after light was switched off under $\operatorname{Ar}\left(1\right.$ bar) and $\mathrm{CO}_{2}(1$ bar). In the blank experiment, no carbon nitride catalyst was added. Conditions: benzylamine $50 \mu \mathrm{mol}, \mathrm{MeCN} 1 \mathrm{~mL}$, time $24 \mathrm{~h}, T=35^{\circ} \mathrm{C}$, gas pressure 1 bar; (c) capacity of K-PHI depending on the benzylamine concentration. Conditions for $\mathrm{K}-\mathrm{PHI}{ }^{\circ-}$ generation: $\mathrm{MeCN} 3 \mathrm{~mL}, \mathrm{CO}_{2} 1$ bar, time $24 \mathrm{~h}, T=35^{\circ} \mathrm{C}$. Conditions for K$\mathrm{PHI}^{--}$quenching: $\mathrm{MV}^{2+} 0.1 \mathrm{mmol}$; (d) Number of electrons ( $y$-axis) transferred from $\mathrm{K}-\mathrm{PHI}{ }^{--}$to $\mathrm{MV}^{2+}$ versus $\mathrm{K}$-PHI amount ( $x$-axis) equal to the number of electrons accumulated in $\mathrm{K}^{-} \mathrm{PHI}{ }^{\cdot-}$. Conditions for $\mathrm{K}-\mathrm{PH}{ }^{\cdot-}$ preparation: initial benzylamine concentration $63 \mu \mathrm{mol} \mathrm{mL}^{-1}$, reaction temperature $35^{\circ} \mathrm{C}$, time $24 \mathrm{~h}, \mathrm{MeCN} 3 \mathrm{~mL}, \mathrm{CO}_{2} 1$ bar. $\mathrm{K}-\mathrm{PHI}{ }^{\circ-}$ quenching conditions: $\mathrm{MV}^{2+} 0.1 \mathrm{mmol}$. See the ESI† for calculation details; (e) EPR spectra. EPR spectra of K-PHI suspension in MeCN in the dark (grey curves) and under $461 \mathrm{~nm}$ light (brown curves) irradiation (BA - benzylamine); (f) quenching of $\mathrm{K}-\mathrm{PHI}{ }^{\cdot-}$ with AgOTf. Reaction was performed under $\mathrm{CO}_{2}$. Appearance of the reaction mixture and schematic representation of the quenching reaction.

distributed in the bulk of the K-PHI particles. These results underline the similarity between the cathode of an electric battery and the K-PHI nanoparticle.

The plot of the number of trapped electrons versus K-PHI mass shows a linear trend, supporting the reproducibility of the method of IDEAS quantification in the range of K-PHI masses up to $10 \mathrm{mg}$ per experiment, which is $787 \pm 35 \mu \mathrm{mol} \mathrm{g}{ }^{-1}$ (Fig. 2d).

Furthermore, we studied the suspension of K-PHI in acetonitrile by EPR (Fig. 2e). In the dark, a broad signal of low intensity at $3350 \mathrm{G}(g=2.016$, after magnetic field calibration) was detected. The intensity of the signal remained almost the same when the EPR spectrum was acquired while the sample was irradiated with $461 \mathrm{~nm}\left(6 \mathrm{~mW} \mathrm{~cm}{ }^{-2}\right)$. Upon addition of benzyl amine to the sample in the dark, the broad signal at 3350 $\mathrm{G}$ disappeared and a narrow signal of low intensity appeared at $3370 \mathrm{G}(g=2.0048)$. Under light irradiation, the intensity of the signal increased drastically supporting that K-PHI* was quenched by benzylamine and the $\mathrm{K}-\mathrm{PHI}^{-}{ }^{-}$radical was formed.

Finally, $\mathrm{K}-\mathrm{PHI}^{--}$can be quenched not only with $\mathrm{MV}^{2+}$, but also with AgOTf, necessarily under oxygen-free conditions. In this case, $\mathrm{Ag}^{+}$is instantly reduced to $\mathrm{Ag}^{\mathrm{O}}$ (Fig. 2f). Formation of $\mathrm{Ag}^{0}$ was confirmed by the presence of (111) and (200) diffraction peaks in the powder X-ray diffraction pattern of the resulting solid (Fig. S6†). Upon the reaction mixture workup, benzylammonium triflate was isolated (Fig. S7†). It suggests that the negative charge of $\mathrm{K}^{-\mathrm{PHI}^{-}}{ }^{-}$is compensated by benzylammonium cations, similar to $\mathrm{K}^{+}$when phosphate buffer is used..$^{18}$ Taking into account the successful quenching of $\mathrm{K}^{-\mathrm{PHI}^{-}}{ }^{-}$with AgOTf and $\mathrm{MV}^{2+}$ and their standard redox potentials, $E\left(\mathrm{Ag} / \mathrm{Ag}^{+}\right)^{0}=+0.80 \mathrm{~V}$ and $E\left(\mathrm{MV}^{2+} / \mathrm{MV}^{++}\right)^{0}=-0.5 \mathrm{~V} v s$. SHE, energetically the SOMO of K-PHI ${ }^{--}$lies at a potential more positive than $-0.5 \mathrm{~V} v s$. SHE.

In order to elucidate the path of benzylamine oxidation, we performed several experiments in $\mathrm{CD}_{3} \mathrm{CN}$ using $\left({ }^{15} \mathrm{~N}\right)$ - 
a)

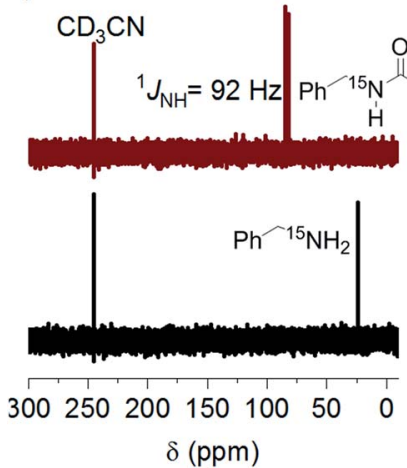

b)

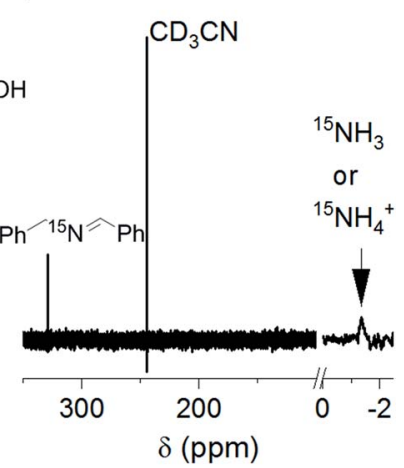

Fig. 3 (a) ${ }^{15} \mathrm{~N}$ NMR spectrum of a $\left({ }^{15} \mathrm{~N}\right)$ benzylamine solution in $\mathrm{CD}_{3} \mathrm{CN}$ : DMSO- $\mathrm{d}_{6}(4: 1)$ before (bottom) and after (top) saturation with $\mathrm{CO}_{2}$; (b) ${ }^{15} \mathrm{~N}$ NMR spectrum of the reaction mixture in $\mathrm{CD}_{3} \mathrm{CN}$ after the photocatalytic experiment. The up-field area was magnified and expanded for clarity.

substituted benzylamine. In excess of $\mathrm{CO}_{2},\left({ }^{15} \mathrm{~N}\right)$ benzylamine is quantitatively converted into benzylcarbamic acid as we deduced from the ${ }^{15} \mathrm{~N},{ }^{1} \mathrm{H}$ and ${ }^{13} \mathrm{C}$ NMR spectra (Fig. 3a). Therefore, benzylcarbamic acid is a more plausible electron donor than benzylamine itself, if the reaction is performed in the presence of $\mathrm{CO}_{2}$. In the ${ }^{15} \mathrm{~N}$ NMR spectrum of the reaction mixture obtained using $\left({ }^{15} \mathrm{~N}\right)$ benzylamine as a substrate, we registered a signal of $\left({ }^{15} \mathrm{~N}\right)$ imine at $329.3 \mathrm{ppm}$ and a broad peak at $-1.4 \mathrm{ppm}$ that corresponds to either ${ }^{15} \mathrm{NH}_{3}$ or ${ }^{15} \mathrm{NH}_{4}{ }^{+}$ (Fig. 3b). In the control experiment using benzylcarbamic acid $(50 \mu \mathrm{mol})$ as an electron donor, we also obtained imine in $40 \%$ yield as evidenced by ${ }^{1} \mathrm{H}$ NMR (Fig. S8†).

The stoichiometry of benzylamine coupling to imine requires evolution of 1 equivalent of $\mathrm{H}_{2}$. However, the amount of $\mathrm{H}_{2}$ detected by GC-TCD (1.48 nmol, Fig. S9†) was substantially lower than that expected from the reaction of benzylamine coupling $(25 \mu \mathrm{mol})$. In order to comply with the mass balance of the reaction, hydrogen is presumably stored in K-PHI. Elemental analysis of the K-PHI after the photocatalytic reaction showed an increased content of hydrogen, $2.60 \pm 0.01 \mathrm{wt} \%$ versus $2.23 \pm 0.03 \mathrm{wt} \%$, in the fresh K-PHI. The increased hydrogen content translates into $18.5 \mu \mathrm{mol}$ of $\mathrm{H}^{+}$(or $9.25 \mu \mathrm{mol}$ of $\mathrm{H}_{2}$ ) that is close to the value obtained from the typical experiment of benzylamine coupling, i.e. $25 \mu \mathrm{mol}$ (reagent loading as shown in Fig. 2b). These results suggest that not only electrons, but also protons from the reaction of benzylamine coupling are stored in K-PHI, which compensate the negative charge. The protons and electrons do not recombine spontaneously, but can be released in the dark by adding a Pt cocatalyst. $^{8}$

\section{Conclusions}

The conditions for the carbon nitride radical anion generation by IDEAS were elaborated, and the number of accumulated electrons was measured by quenching the carbon nitride radical anion with a common redox indicator - methylviologen dichloride. This method is generally applicable for the characterization of semiconducting materials regarding their ability to accumulate electrons. Carbon nitride materials can be charged up to at least $957 \pm 82 \mu \mathrm{mol} \mathrm{g}^{-1}\left(92 \pm 8 \mathrm{C} \mathrm{g}^{-1}\right)$ using benzylamine as a convenient electron donor in the presence of $\mathrm{CO}_{2}$. Every $4^{\text {th }}$ heptazine unit in K-PHI thus holds an uncoupled electron. Optimization of the poly(heptazine imide) structure toward a higher capacity by IDEAS is one of the strategies to enhance further the photocatalytic performance of the material. Selection of stronger electron donors can also potentially increase the density of accumulated electrons in semiconductors.

\section{Conflicts of interest}

There are no conflicts to declare.

\section{Acknowledgements}

The authors are grateful to the Deutsche Forschungsgemeinschaft for the financial support (DFG-An 156 131). PL and NL acknowledge the Danish National Research Foundation (DNRF 118, CADIAC). PL, NL and JC thank the AU Synergic grant for partially supporting their work.

\section{References}

1 L. Marzo, S. K. Pagire, O. Reiser and B. König, Angew. Chem., Int. Ed., 2018, 57, 10034-10072.

2 I. K. Sideri, E. Voutyritsa and C. G. Kokotos, Org. Biomol. Chem., 2018, 16, 4596-4614.

3 D. P. Hari and B. König, Chem. Commun., 2014, 50, 66886699.

4 A. Savateev, M. Antonietti, I. Ghosh and B. König, Angew. Chem., Int. Ed., 2018, 57, 15936-15947.

5 A. Savateev and M. Antonietti, ACS Catal., 2018, 8, 97909808.

6 I. Ghosh, J. Khamarai, A. Savateev, N. Shlapakov, M. Antonietti and B. König, Science, 2019, 365, 360-366.

7 G. Zhou, Y. Shan, Y. Hu, X. Xu, L. Long, J. Zhang, J. Dai, J. Guo, J. Shen, S. Li, L. Liu and X. Wu, Nat. Commun., 2018, 9, 3366.

8 V. W. h. Lau, D. Klose, H. Kasap, F. Podjaski, M. C. Pignié, E. Reisner, G. Jeschke and B. V. Lotsch, Angew. Chem., Int. Ed., 2016, 56, 510-514.

9 A. Savateev, B. Kurpil, A. Mishchenko, G. Zhang and M. Antonietti, Chem. Sci., 2018, 9, 3584-3591.

10 B. Kurpil, Y. Markushyna and A. Savateev, ACS Catal., 2019, 9, 1531-1538.

11 H. Ou, C. Tang, X. Chen, M. Zhou and X. Wang, ACS Catal., 2019, 9, 2949-2955.

12 H. Schlomberg, F. Podjaski, J. Kroeger and B. Lotsch, Nachr. Chem., 2018, 66, 1157-1161.

13 W. Yang, R. Godin, H. Kasap, B. Moss, Y. Dong, S. A. J. Hillman, L. Steier, E. Reisner and J. R. Durrant, J. Am. Chem. Soc., 2019, 141, 11219-11229. 
14 H. Kasap, C. A. Caputo, B. C. M. Martindale, R. Godin, V. W.-h. Lau, B. V. Lotsch, J. R. Durrant and E. Reisner, J. Am. Chem. Soc., 2016, 138, 9183-9192.

15 B. Kurpil, K. Otte, A. Mishchenko, P. Lamagni, W. Lipiński, N. Lock, M. Antonietti and A. Savateev, Nat. Commun., 2019, 10, 945.

16 Y. Xu, X. He, H. Zhong, D. J. Singh, L. Zhang and R. Wang, Appl. Catal., B, 2019, 246, 349-355.

17 Z. Zeng, X. Quan, H. Yu, S. Chen, Y. Zhang, H. Zhao and S. Zhang, Appl. Catal., B, 2018, 236, 99-106.

18 F. Podjaski, J. Kröger and B. V. Lotsch, Adv. Mater., 2018, 30, 1705477.

19 B. Kurpil, B. Kumru, T. Heil, M. Antonietti and A. Savateev, Green Chem., 2018, 20, 838-842.
20 A. Savateev, D. Dontsova, B. Kurpil and M. Antonietti, J. Catal., 2017, 350, 203-211.

21 H. Schlomberg, J. Kröger, G. Savasci, M. W. Terban, S. Bette, I. Moudrakovski, V. Duppel, F. Podjaski, R. Siegel, J. Senker, R. E. Dinnebier, C. Ochsenfeld and B. V. Lotsch, Chem. Mater., 2019, 31, 7478-7486.

22 B. Kurpil, A. Savateev, V. Papaefthimiou, S. Zafeiratos, T. Heil, S. Özenler, D. Dontsova and M. Antonietti, Appl. Catal., B, 2017, 217, 622-628.

23 Z. Chen, A. Savateev, S. Pronkin, V. Papaefthimiou, C. Wolff, M. G. Willinger, E. Willinger, D. Neher, M. Antonietti and D. Dontsova, Adv. Mater., 2017, 29, 1700555. 